\title{
Die Resultate der zoologischen Erforschung hochalpiner Wasserbecken seit dem Jahre 1900.
}

\author{
Von \\ Prof. F. Zschokke (Basel). \\ Literaturverzeichnis.
}

1) Brehm., V., 1902, Zusammensetzung, Verteilung und Periodizität des Zooplanktion im Achensee. Zeitschr. des Ferdinandeums, III, S. 46.

2) Brehm, V., 1905, Zur Besiedelungsgeschichte alpiner Seebecken. 77. Versamml. deutscher Naturf. u. Ärzte in Meran.

3) Brehm, V., 1905, Untersuchungen über das Zooplankton einiger Seen der nördlichen und östlichen Alpen. Verhandl, der k. k. zoolog.-botan. Gesellschaft in Wien, (Jahrg. 1906).

4) Brehm, Y., 1907, Die biologische Süßwasserstation zu Lunz-Seehof, Niederösterreich. Archiv f. Hydrobiologie u. Planktonkunde, II, 1907.

5) Brehm, V., 1907, Uber das Vorkommen von Diaptomus tatricus Wierz. in dén Ostalpen und über Diaptomus Kupelwieseri. n. sp. Zool. Anz., Bd. XXXI, 1907.

6) Brehm, V., Beiträge zur faunistischen Durchforschung der Seen Nordtirols. 1907.

7) Brehm, V. und Zederbauer, E., 1904, Beiträge zur Planktonuntersuchung alpiner Seen I. Verhandl, d. zoolog.-botan. Gesellschaft in Wien, 1904.

8) Brehm, V. und Zederbauer, E., 1904, Beiträge zur Planktonuntersuchung alpiner Seen II. Ebenda 1904.

9) Brehm, V. und Zederbauer, E., 1905, Beiträge zur Planktonuntersuchung etc. III. Ebenda 1905.

10) Brehm, V. und Zederbauer, E., 1906, Beiträge zur Planktonuntersuchung etc. IV. Ebenda 1906.

11) Brehm, V. und Zederbauer, E., 1906, Beobachtungen über das Plankton in den Seen der 0stalpen. Archiv f. Hydrobiologie u. Planktonkunde, I, 1906.

12) Bretscher, K., 1900, Mitteilungen über die Oligochätenfauna der Schweiz. Revue Suisse de zoologie, 1900, T. VIII. 
13) Bretscher, K., 1901, Beobachtungen über Oligochäten der Schweiz. Ebenda 1901, T. IX.

14) Bretscher, K., 1902 , Beobachtungen über Oligochäten der Schweiz. VI. Ebenda 1902, T. X.

15) Bretscher, K., 1903, Beobachtungen über Oligochäten der Schweiz. VII. Ebenda 1903, T. XI.

16) Bretscher, K., 1905, Beobachtungen über oligochäten der Schweiz. IX. Ebenda 1905, T. XIII.

17) Buffa, P., 1902, Sulle Condizioni fisiche e biologiche di taluni laghi alpini del Trentino. Atti della Società Veneto-Trentina di Scienze naturali T. IV. 2, 1902.

18) Ekman, Sven, Die Phyllopoden, Cladoceren und freilebenden Copepoden der nordschwedischen Hochgebirge. Zool. Jahrb. Abt. Syst. Geograph. Biol., Bd, XXI, 1904.

19) v. Hofsten, Nils, 1907, Studien über Turbellarien aus dem Berner Oberland. Zeitschr. f. wissensch. Zool., Bd. LXXXV, 3 u. 4, 1907.

20) Keilhack, L., 1906, Cladoceren aus den Dauphinéalpen. Zool. Anz. Bd. XXIX, 1906.

21) Könike, F., 1902, Acht neue Lebertia-Arten, eine neue Arrenurusund eine Atractides-Art. Zool. Anz., Bd. XXV, 1902.

22) Monti, Rina, 1903, Le Condizioni fisico-biologiche dei laghi Ossolani e Valdostani in rapporto alla piscicoltura. Memoria letta al reale Istituto Lombardo 1903.

23) Monti, Rina, 1904, Limnologische Untersuchungen über einige italienische Alpenseen. Forschungsberichte aus der biologischen Station zu Plön, XI, 1904.

24) Monti, Rina, 1904, Physiobiologische Beobachtungen an den Alpenseen zwischen dem Vigezzo- und dem Onsernonetal. (1904.) Ebenda, $\mathrm{XI}, 1904$.

25) Monti, Rina, 1905, Un modo di migrazione del plancton finqui sconosciuto. Rendiconti del R. Ist. Lombard. di sc. e lett., Serie II. vol. XXXVIII, 1905.

26) Monti, Rina, 1906, Recherches sur quelques lacs du massif du Ruitor. Annales de biologie lacustre, I, 1906.

27) Monti, Rina, 1906(07) La circolazione della vita nei laghi. Rivista mensile di pesca, IX, 1907.

28) Penard, E., 1905, Sarcodinés. Catalogue des Invertébrés de la Suisse. Genève 1905.

29) Steinmann, P., 1906, Geographisches und Biologisches von Gebirgsbach-Planarien. Archiv f. Hydrobiol. u. Planktonkunde. Bd. II, 1906.

30) Steuer, A., Die Entomostrakenfauna der alten Donau bei Wien. Eine ethologische Studie. Mit einem Anhang: Zur Frage über Ursprung und Verbreitung der Entomostrakenfauna des Süßwassers. Zool. Jahr. Abt. Syst. Geograph. Biol., Bd. XV. 1901.

31) Stingelin, Th., 1904, Die Familie der Holopedidae. Revue suisse de Zool., Bd. XII, 1904.

32) Stingelin, Th., 1906, Neue Beiträge zur Cladocerenfauna der Schweiz. Ebenda, Bd. XIV, 1906.

33) Thor, Sig, 1905, Neue Beiträge zur schweizerischen Acarinenfauna. Revue Suisse de Zoologie. Bd. XIII. 1905. 
34) Walter, C., 1907, Die Hydracarinen der Schweiz. Revue Suisse de Zoologie, Vol. XV, 1907.

35) Woltereck, R., 1906, Mitteilungen aus der biologischen Station in Lunz (N.-0.). Biolog. Zentralblatt, Bd. XXVI, 1906.

36) Zoltán v. Szilády, 1900, Die Crustaceen des Retyezát. Mathemat. u. naturwissenschaftl, Berichte aus Ungarn. XVIII, 1900.

37) Zschokke, F., 1907, Rana fusca Rösel und Triton alpestris Laur. als Bewohner der Hochalpen. Wochenschr., IV, 1907,

38) Zschokke. F., 1900, Die Tierwelt der Hochgebirgsseen. Neue Denkschriften der schweizerischen naturforschenden Gesellschaft 1900.

Die zahlreichen durch die Hochalpen ausgestreuten stehenden Gewässer, die nahe Gipfel und Kamm oberhalb der Baumgrenze liegen, beanspruchen faunistisch, biologisch und tiergeographisch ein ganz besonderes Interesse. Ihr äußerer Charakter wechselt von Ort zu Ort in bunter Folge. Bald sind es in Felskessel gebettete oder von öden Geröllhalden begrenzte Becken; bald ruhen die Seen inmitten reich bewachsener Alpweiden. Andere Wasserbehälter liegen an der Gletscherwand und am nie ganz schmelzenden Schneefeld, auf ihren Spiegeln schwimmen ungefüge Eisblöcke. Wieder andere Gewässer stellen seichte, austrocknende oder beständige Tümpel dar, die jeder warme Tag überhitzt, jede folgende kalte Nacht mit einer Eiskruste überspannt.

Mit dem Bild der äußeren Bedingungen wechselt die faunistische Zusammensetzung der Bewohnerschaft. Im ganzen aber finden sich in der Tierwelt hochalpiner Seebecken zwei Elemente zusammen: eurytherme, gegen äußere Verhältnisse sehr resistente Kosmopoliten und stenotherme Kaltwasserbewohner mit nordischem oder glazialem Charakter und von beschränktem Verbreitungsbezirk. Die letztgenannten Tiere stellen wahrscheinlich Oberreste einer früher weitverbreiten Eiszeitfauna dar; sie folgten am Schluß der allgemeinen Vereisung den weichenden Gletschern in die Alpen und fristen in hochgelegenen Wasserbehältern, die noch heute unter glazialarktischen Bedingungen stehen, ein isoliertes Dasein. Ihren. Zusammenhang mit den von ihnen heute getrennten Verwandten des hohen Nordens systematisch, geographisch und biologisch zu erkennen, den durch Anpassung an hochalpine Verhältnisse hervorgerufenen Veränderungen in Bau, Lebensweise und Fortpflanzung nachzugehen, den morphologischen und biologischen Jahreszyklus der Bewohner von Hochgebirgsgewässern festzustellen, bildet ein vornehmes Ziel der Erforschung hochalpiner Seen.

Seit der Referent im Jahre 1900 sein zusammenfassendes Werk über die Tierwelt der Hochgebirgsseen veröffentlichte, ruhte die Untersuchung der Alpenseen nicht, wenn sie auch hin und wieder ihr Endziel aus den Augen verlor und umsichtige Kritik vermissen ließ.

Eine erwünschte Ausdehnung des Untersuchungsgebietes, das sich bisher vornehmlich auf den Nordhang der Alpen und besonders die Schweiz beschränkt hatte, brachten die Forschungen von R. Monti. Die Fänge der eifrigen, italienischen Zoologin erstrecken sich über eine große Anzahl sehr verschiedenartiger Hochgebirgsseen der Südalpen. Es fallen in den erforschten Bezirk die bis gegen $2600 \mathrm{~m}$ Meereshöhe liegenden Seen des Aosta- und Tosatals, die Gletscherbecken des mächtigen Ruitorstocks in den Grajischen Alpen, an der Grenze von Piemont und Savoyen, in den Höhenlagen von 2000 bis $2600 \mathrm{~m}$, ein Hochsee am Monte Rosa, der Mattmarksee an der Moräne des Allalingletschers und end- 
lich die zwischen Vigezzo- und Onsernonetal an der Tessinergrenze bei 1900 bis $2050 \mathrm{~m}$ gelegenen Wasseransammlungen.

Einige faunistische Bereicherung der Kenntnisse über die Tierwelt südalpiner Hochseen brachten auch die Forschungen Buffas im Trentino.

Die Untersuchungen über das Plankton subalpiner Seen der 0stalpen, deren Ergebnisse so bemerkenswerte Schlüsse über Tiergeographie und postglaziale Besiedelungsgeschichte zeitigten, führten Brehm auch in die hochalpine Zone, zunächst im Nordtirol, dann in den Dolomiten, den hohen Tauern und endlich zu den Almtümpeln bei Lunz in Niederösterreich. Der genannte Forscher besuchte vier Seen im Sellrain (2200 bis $2300 \mathrm{~m}$ Höhenlage), den Lauterersee im Gschnitztal $(2400 \mathrm{~m})$, der erst im September auffiert, um schon einen Monat später wieder seine Eisdecke zu erhalten, den in derselben Gegend gelegenen Lichtsee $(2200 \mathrm{~m})$, die Becken am Pfitscher Joch und den Schwarzsee bei der Berliner Hütte im Zillertal. In das Gebiet der Dolomiten fallen der Dürrensee, der Pragser Wildsee und der Misurinasee $(1755 \mathrm{~m})$, von denen indessen wenigstens die beiden ersten kaum der hochalpinen Region angehören. In den Tauern veranstaltete Brehm Fänge am Sonnblick (Pockhardsee $1825 \mathrm{~m}$ ).

Noch weiter noch Osten greifen die faunistisch-biologischen Untersuchungen über die Crustaceen des Retyezattgebirgs in den transsylvanischen Alpen aus. Szilady befischte dort vier Kategorien hochgelegener Wasseransammlungen, von denen die beiden letzten, Torfgewässer und Hochgebirgseen, genetisch in enger Beziehung zur ehemaligen Vergletscherung stehen.

Den hochalpinen Cladoceren widmete Stingelin wiederholt durch systematische und faunistische Gründlichkeit ausgezeichnete Kapitel.

Endlich finden sich Notizen über die Fauna hochgelegener Wasserbecken eingestreut in Arbeiten, deren Hauptziel in anderer Richtung liegt. So berichtet Penard gelegentlich über Rhizopoden, v. Hofsten und Steinmann über Turbellarien, Keilhack über Cladoceren, Bretscher über Oligochäten, Sig Thor und Walter über Hydrachniden der in diesem Referat in Betracht fallenden Region, d. h. der stehenden Gewässer oberhalb der Grenze zusammenhängenden Waldes.

Im ganzen macht der Gang der zoologischen Erschließung hochalpiner Seen im Laufe der letzten Jahre den Eindruck extensiver Ausdehnung über weite geographische Gebiete oft auf Kosten intensiverer Vertiefung der Studien.

Es liegt in der Natur der Sache begründet, in den zahlreichen lokalen Schwierigkeiten, die sich einer über längere Zeiträume ausgedehnten Untersuchung im Hochgebirge entgegenstellen, daß vor allem die reine Faunistik aus den Beobachtungen Nutzen zog, Tiergeographie und Biologie aber sich mit viel bescheideneren Erfolgen begnügen mußten. So erfuhr das allgemeine Bild der Tierwelt von Hochalpenseen, wenn nicht eine prinzipielle Veränderung oder Verschiebung, so doch eine nicht unbedeutende Erweiterung und Ergänzung. Besonders auf das Vordringen eurythermer Kosmopoliten bis in alpine Gewässer von beträchtlicher Höhenlage fiel manches neue Licht. Es mag vollauf genügen, aus dem reichen Material einige wenige Daten in derselben systematischen Folge, wie im Werk über die Tierwelt der Hochgebirgseen, anzuführen.

Die Liste der hochalpinen Rhizopoden vergrößerte sich nicht unbeträchtlich durch die Untersuchungen Montis und Penards. Von einigem Interesse mag es sein zu erfahren, daß Difflugia bacillifera Penard, Nebela bigibbosa Penard und Amphitrema stenostoma Nüßlin im Lac des Conches bei $1700 \mathrm{~m}$ Höhenlage leben (Wallis). Hyalosphenia lata F. E. Schulze steigt im Vigezzo- 
Onsernonegebiet bis gegen $2000 \mathrm{~m}$, und Difflugia urceolata Carter verbreitet sich vertikal auf dem Großen St. Bernhard bis zu $2467 \mathrm{~m}$, im Lago Licone des Aostatals bis $2553 \mathrm{~m}$.

An ihren maximalen Höhengrenzen stehen, nach den bis heute vorliegenden Daten, Paramaecium aurelia 0. F. M., Ophryoglena griseo-virens Perty und Glaucoma scintillans Ehrbg. im kleinen See auf der Jochhöhe des Großen St. Bernhard, der vom nahegelegenen Hospiz aus eine reiche Zufuhr faulender, organischer Substanzen erhält.

In seiner Ausbeute rhabdocoeler Turbellarien aus dem Berner oberland zäblt v. Hofsten dreizehn hochalpine Arten auf. Am ergiebigsten waren kleine, warme Teiche, arm die größeren, kalten Seen. Bis gegen $2300 \mathrm{~m}$ erheben sich Rhynchomesostoma rostratum (Müll.), Mesostoma lingua Abildg., Castrella truncata Abildg., Gyratrix hermaphroditus Ehrbg., Castrada affinis n. sp., Castrada luteola n. sp., Dalyellia diadema n. sp.

Auch im Ruitormassiv kennzeichnet Mesostoma lingua hochgelegene Wasserbehälter; die Turbellarie macht einen typischen Bestandteil der Fauna der sich überhitzenden Almtümpel und "Blutseen" aus.

Unter den Tricladen herrscht in der oberen Waldregion und in der hochalpinen Zone des Berner Oberlandes unbeschränkt Planaria alpina; dasselbe typische Glazialtier fand R. Monti am Ruitor und in den Gewässern des Aostaund Tosatals regelmäBig bis zu $2600 \mathrm{~m}$. In hohem Grade auffallend klingt die Angabe, daß im Lago Zyole, in der bedeutenden Höhe von $2521 \mathrm{~m}$, die Alpenplanarie fehle. Neben dem nordischen Diaptomus bacillifer und der stenothermen Kaltwasserbewohnerin Polycelis cornuta sollen in dem genannten Gewässer die Tricladen der warmen Teiche und Bäche der Ebene Dendrocoelum lacteum, Planaria gonocephala und Polycelis nigra, die höhere Gebirgslagen sonst vollständig meiden, vorkommen. Etwas oberhalb des Sees lebt dagegen in Quellen wieder Pl. alpina.

Sollte eine erneute, dringend erwünschte Kontrolle die Gegenwart der Flachlanddendrocoelen im Lago Zyole bestätigen, so würden die in zahlreichen, sorgfältigen Arbeiten besprochenen und niedergelegten Ansichten über die postglazialen Wanderungen und gegenseitigen Verdrängungen der Süßwassertrikladen eine weitgehende Modifikation erfahren müssen. Die Vorstellungen über die Geschichte der niederen Süßwasserbewohner im Anschluß an die Gletscherzeit wären in manchen Punkten zu ändern!

Notholca longispina Kellic. behauptet auch nach den neueren Untersuchungen die führende Stellung unter den Planktonrotatorien hochalpiner Seen, das gilt für Nord- und Sültirol; am Ruitor tritt bei $2402 \mathrm{~m} \mathrm{~N}$. striata an ihre Stelle. Die Seen der Trientiner Alpen beherbergen, neben N. Iongispina und Anuraea aculeata, Polyarthra platyptera und Notholca labis (Lago delle Stellune $2140 \mathrm{~m}$ und Lago Lagorai $1850 \mathrm{~m}$ ). Auch Polyarthra platyptera spielt im Plankton der Hochalpen nach Häufigkeit und Verbreitung eine große Rolle. Sie lebt sowohl im Sellrain- und Gschnitztal in Tirol, als in den Tauern und in Hochseen der grajischen Alpen. Triarthra longiseta überschreitet die Grenze von $2200 \mathrm{~m}$ beträchtlich in den Tiroler Alpen. Dort und in den hohen Tauern entfalten sich Anuraea aculeata, Triarthra longiseta und Polyarthra platyptera an hochgelegenen Fundorten $\mathrm{zu}$ im Tiefland nicht erreichter Individuenzahl. Oberhaupt konnte Brehm feststellen, daß die Rotatorien in der eigentlichen hochalpinen Region das Plankton an Menge beherrschen. Endlich rücken neu in die Liste der hochalpinen Rädertierchen ein Gastropus styRevue d. ges. Hydrobiol, a. Hydrogr. Bd, I. II. 1 u. 2. 
lifer Imhof (Roggia-Alpsee $1920 \mathrm{~m}$ ) und Philodina tuberculata (See von Panelatte $2048 \mathrm{~m}$ ).

Ungemein erweitert wurdeu durch die sorgfältigen Untersuchungen Bretschers die Kenntnisse über das Vorkommen und die Verbreitung der limikolen Oligochäten am Nordhang der zentralen und östlichen Schweizeralpen. Wieder genießen die in der Ebene überall lebenden Formen auch im Gebirge horizontal und vertikal die ausgiebigste Verbreitung; sie bevölkern sogar kleine Wassclansammlungen von ausgesprochen glazialen Gepräge. Der höchstgelegene Fundort, ein seichter Trümmersee auf der Göscheneralp $(2300 \mathrm{~m})$, lieferte noch zwei Arten der Gattung Mesenchytraeus, M. bisetosus Br. und M. alpinus Br.

Manche neue Bestätigung liegt auch vor für das häufige und massenhafte Auftreten kosmopolitischer Ostracoden, Cyclopiden und Harpacticiden bis auf Gebirgsstufen von sehr bedeutender Erhebung und bis an den Gletseherrand.

Seine Erfahrungen über das Vorkommen von Cladoceren in hochalpinen Gewässern faßt stingelin dahin zusammen, daß allgemeine Verbreitung genießen Daphnia longispina, Acroperus harpae, Alona affinis, Alonella excisa und Chydorus sphaericus. Seltener treten auf Holopedium gibberum, Scapholeberis mueronata, Ceriodaphnia quadrangula, Macrothrix hirsuticornis, Alona quadrangula, A. costata, A. guttata, selir selten Ceriodaphnia pulchella, Moina micrura, Bosmina obtusirostris, Alona intermedia, Alonella exigua und Peracantha truncata. Nach R. Montis Zusanmenstellungen wäre noch das nicht seltene Auftreten von Sida crystallina und Alona rostrata im Gebiet der Tosa und von Aosta zu nennen; beide Krebse bevölkern den See von Licone, ein Wasserbecken von skandinavischem Gepräge $(2553 \mathrm{~m})$ und von hochalpinem faunistischem Charakter. Mit ilnen teilt den Wohnort Crepidocercus setiger, Planaria alpina und der Wasserkäfer Helophorus glacialis. Das Plankton des Zirmsees $(2500 \mathrm{~m})$, dessen Ufer am 18. August noch unter einer tiefen Schneedecke ruhten, sctzte sich, wie Brehm berichtet, aus dem Ubiquisten Chydorus sphaericus begleitet von dem typischen Glazial-Copepoden Cyclops strenuus zusammen.

Sollen außer den schon genannten Arten noch einige für die Hochalpen neue Cladoceren aufgezählt werden, so mögen Erwähnung finden Alonella nana (Danphiné bei .ca. $2000 \mathrm{~m}$ Meereshöhe), Alona rectangula aus dem Bettmerspe im Wallis $(1991 \mathrm{~m})$, A. leydigii und Moina brachiata aus den Bergen des Vigezzo- und Onsernonetals (ca. $1900 \mathrm{~m}$ ), und Chydorus sphaericus var. caelatus vom St. Gothlard.

Für das sporadische Auftreten von Gammar us pulex in höheren Gebirgslagen spricht ein Fund von Szilády, der den gewöhnlichen Flohkrebs in einem Hochsee der transsylvanischen Alpen bei $1809 \mathrm{~m}$ antraf.

Eigentümlich ist es, daß die Hydrachnidenfunde in Hochgebirgsseen ausschließlich eurytherme auch im Warmwasser des Flachlands verbreitete Formen betreffen. Thor meldet aus zentralschweizerischen Alpenseen von $2000 \mathrm{~m}$ Höhenlage Curvipes carneus (Koch). Montis Lebertia pavesii aus dem Kastelsee $(2215 \mathrm{~m})$ bestimmt Thor richtiger als die in den Alpen nicht selten, aber auch im Flachland vorkommende L. rufipes Könike. Statt der früher unrichtig benannten Lebertia tau-insignita kennt Könike nunmehr aus den Rhätikonseen drei auch im Seichtwasser der Ebene lebende Lebertia-Arten (L. cognata Könike, L. subtilis Könike auch L. rufipes (Könike). Auch die von Walter bestimmten pelagischen Hydrachnidennymphen aus dem Heidsee in Graubünden 
zählen zu keinen nordisch-glazialen Genera. Dasselbe gilt von der alpin etwa auftretenden Piona disparilis.

Unter wie extremen Bedingungen Hydracarinen leben können, zeigt der Fund Stingelins von zahlreichen Milbenlarven im eiskalten Wasser des vom Aletschgletscher begrenzten Märielensee.

So stellt sich die Hydrachnidenfauna der Hochgebirgsseen in einen scharfen Gegensatz zu derjenigen der kalten und schäumenden Bergbäche. In den letztgenannten Gewässern fand die neuere Forschung eine lange Reihe arktisch-alpiner Wassermilben. Der See erhielt durch passiven Import seit dem Rückzug der Gletscher kosmopolitische Hydracarinen, in den Bach zogen sich aktiv die Trümmer einer stenothermen Schmelzwasserfauna zurück.

Uber die Verbreitung von Forellen und Saiblingen, von Cottus gobio und Phoxinus laevis, und endlich von Rana fusca und Triton alpestris in den Hochalpen liegen einzelne zertreute Notizen vor. Zschokke stellt neue und alte Daten über Fortpflanzung und Metamorphose der beiden hoch in die Alpen emporsteigenden Amphibien zusammen.

In den vorhergehenden Zeilen lag wiederholt der Hinweis darauf, daß die neueren Forschungen auch über Vorkommen und Verbreitung der arktisch-alpinen Kaltwasserbewohner im Gebirge mancherlei Licht verbreitet haben.

Mit aller Vorsicht rechnet Bretscher den gegen Kälte so äuBerst resistenten oligochaeten Henl ea vertriculos a hierher, dessen Vorkommen in Patagonien, Neuseeland und Chile er durch Verschleppung erklärt. Vielleicht reiht sich hier auch Castrada sphagnorum aus Finnland an; v. Hofsten fand die Turbellarie in Hochtümpeln des Berner Oberlandes wieder.

Für die arktischen Copepoden Cyclops strenuus, Diaptomus bacillifer und D. denticornis ergeben sich eine ganze Reihe neuer, hochalpiner Fundorte. D. denticornis lebt, nach Stingelin, auf dem St. Gotthard, nach Brehm, in den Hochseen der Tiroler Alpen, wo D. bacillifer fehlt; er tritt regelmäBig in den Becken der Gebirge von Aosta und Domo d'Ossola auf. Seinen höchsten dortigen Wohnort, den Zyolesee $(2521 \mathrm{~m})$, soll er sogar mit D. bacillifer teilen, ein sonst nicht mit Sicherheit beobachtetes Vorkommnis. Mehr sporadisch erhebt sich in einzelnen vorgeschobenen Kolonien der ebenfalls nordische D. gracilis in das Hochgebirge; im Lago Vannino der Südalpen stellt er sich zugleich mit D. denticornis ein. Brehm nennt den Calaniden den typischen pelagischen Diaptomus der tiefer liegenden Seen der Ostalpen. Von Diaptomus laciniatus entdeckte Steuer einen Standort auf der Saualpe in Kärnten $(2000 \mathrm{~m})$. Damit erhielt der arktische Centripagide, der die schweizerischen, subalpinen Randseen, die Gebirgsbecken der Pyrenäen und Gewässer des Schwarzwaldes, der Auvergne und des französischen Juras bevölkert, auch eine bescheidene Vertretung in der baumlosen Zone der Hochalpen.

Das Verbreitungsbild der hochnordischen Diaptomiden in den großen zentraleuropäischen Gebirgen ergänzt sich durch das Auftreten von D. bacillifer in den Glazialseen des Retyezat bis zu $2200 \mathrm{~m}$. Doch darf nicht verschwiegen werden, daß der Krebs auch in den Pusztagewässern Ungarns weit verbreitet vorkommt.

In den kleinen und seichten Alpseen des Vigezzo-0nsernonetals traf Monti große Mengen durch Carotine hochrot gefärbte Exemplare von Heterocope saliens und bestätigte so eine viel früher von Imhof in hochgelegenen Becken des Kantons Graubünden gemachte Entdeckung. H. saliens gehört der Tierwelt nordischer Seen, Teiche und Tümpel an. In Zentraleuropa lebt das Tier vorzugsweise, 
wenn auch nicht ausschließlich, limmetisch im Plankton der großen Seen. Erst in den Hochalpen stellt es sich wieder, wie an den Grenzen der Arktis, in Kleingewässern ein. Den Import von Heterocope in die Gebirgsseen von Pisola, Panelatte und der Roggiaalpe denkt sich Monti vermittelt durch vom Lago magrgiore herziehende Wasservögel.

Deutlich nordischen Charakter trägt nach seiner geographischen Verbreitung Holopedium gibberum, dessen Kolonie im Herzen der Zentralalpen, in den Hochseen und Tümpeln der St. Gotthardgruppe, Stingelin faunistisch und biologisch näher studierte. Nach der Gletscherzeit war der Krebs mit manchen Verwandten in die kalkarmen Hochgebirge zurückgedrängt worden, um sich in der Ebene nur ausnahmsweise, besonders etwa pelagisch in größeren Seen, zu halten. Auch die Bosmina des Lucendrosees $(2083 \mathrm{~m}$ ) bestimmt $\mathrm{Stingelin}$, als die nordische B. obtusirostris, dabei läßt er allerdings die Frage offen, ob es sich um die forma arctica handle. Es mag an dieser Stelle erwähnt werden, daß die in den skandinavischen Alpen so ausgiebig verbreiteten Bosminen auch in den Alpen reichere Vertretung finden, als man nach früheren Untersuchungen anzunehmen geneigt war. Im arktischen Lautertrsee im Gschnitztal (2400 m Tirol) lebt Bosmina longirostris, im Misurinasee B. cornuta.

$\mathrm{Zu}$ den nordischen Cladoceren, deren Gegenwart im mitteleuropäischen Hochgebirge erst jüngst nachgewiesen wurde, zählt auch die skandinavische und finnische Alona intermedia Sars. Stingelin stellte ihre Gegenwart für die Gotthardseen fest. Dieselbe Form steigt im Retyezat bis $1866 \mathrm{~m}$. Interesse erweckt Stingelins Entdeckung von Acroperus harpae var. frigida Ekman in Gesellschaft des Ubiquisten und Kosmopoliten Chydorus sphaericus im Märjelensee $(2367 \mathrm{~m})$, in dessen stets tief temperiertes Wasser die Wand des Aletschgletschers abstürzt. Die Cladocere bildet einen typischen Bestandteil der Fauna der Flechtenregion Nordskandinaviens; in der Grauweidenregion nähert sie sich durch Zwischenformen der reinen Stammart. Dieselben Ubergänge fand Stingelin in den Seen und Tümpeln des St. Gotthard (2000-2250 m), die er in zwei verschiedenen Jahren zum Gegenstand seiner faunistischen Untersuchungen nachte. Der hoclmordische Charakter von $A$. harpae-frigida tritt noch klarer hervor dureh die Feststellung, daß die Varietät bei Berlin als Winterform erscheint und auch in Berggewässern der Dauphiné und der Tatra lebt.

Den postglazialen nordischen Relikten im Alpengebiet reiht die Tiergeographie auch die Genera Sida und Polyphemus an. Uber letzteren sammelte Keilhack im Hochgebirge der Dauphiné wichtige Daten. Er fand Polyphemus pediculus in Seen von $2000 \mathrm{~m}$ Höhenlage und verfolgte auch Ende Juli in einem der Gebirgsgewässer das Auftreten der Männchen und die Bildung der Wintereier. Die Geschlechtsperiode beschränkt sich bei der hochalpinen Polyphemus-Kolonie auf eine Generation, wie das $\mathrm{Ekman}$ auch für Nordschweden feststellte. Immerhin zieht sich in den Alpen die Zeit zweigeschlechtlicher Fortpflanzung üler einen längeren Zeitraum aus, als in Skandinavien. Der ganze alpine Zyklus mag sich etwa über $1 \frac{1}{2}$ Monate erstrecken. Für Keilhack wich Polyphemus postglazial mit dem Eisrand in die Alpen zurück. Im Hochland und in seiner ursprünglichen nordskandinavischen Heimat behielt der Krebs den arktischen Jahreszyklus bei, während er es verstand, seine Generationenfolge in den Gewässem der zwischenliegenden Ebene den veränderten Bedingungen anzupassen.

Brehm möchte Polyphemus, in Ubereinstimmung mit Ekman, als nordöstlichen Einwanderer in das Gehiet der Alpen betrachten. Immerhin zeigen 
Keilhacks faunistische Befunde, daB sich der Krebs im Hochgebirge weit nach Westen verbreitet.

An der östlichen Abdachung der Alpen findet Brehm die Fauna um südöstliche Interglazialrelikte bereichert (Asplanchna syrinx, Diaptomus zachariasi). Diaptomus tatricus, der in den Karpaten herrscht und im Retyezatgebirge Torfgewässer, Lehmteiche und Hochgebirgsseen bis zu $2000 \mathrm{~m}$ bevölkert, besetzt als nordwestlichste Station die Lunzer Almtümpel. Brehm beansprucht das Tier als östlichen Zuwanderer, dessen Vorfahren ihren Wohnort einst weit nach Westen ausdehnten, später aber durch den von Nordwesten einwandernden, glazialen D. laciniatus nach 0sten zurückgedrängt, am 0strand der Alpen nur noch vereinzelte Standorte zu halten vermochten. Dagegen gilt Ekman D. tatricus mehr als endemische Form der Karpathen.

Brehms Annahme, daß die den Eiszeiten folgende xerotherme Versteppung. den Alpenländern östliche Zuwanderer brachte, die heute in isolierten Kolonien weiterleben und vielfach den Weg der Varietätenbildung beschritten haben, gewinnt an Wahrscheinlichkeit durch die Betrachtung der terrestrischen Fauna. Auch in ihr deuten gewisse aus Westen, Süden und Osten stammende Elemente daraufhin, daß dem Rückzug der Gletscher ein trockenes und warmes Steppenklima folgte. Der heterogene Ursprung der hochalpinen Tierwelt aus räumlich und zeitlich verschiedenartigen Quellen findet, wie die kurzen vorhergehenden Notizen zeigten, in den neueren Forschungen schlagende Beweise.

Allen Beobachtern fiel immer wieder die faunistische Verschiedenheit einander oft sehr naheliegender Hochgebirgsseen auf.. Sie prägt sich sowohl qualitativ in verschiedener Artenzahl, als quantitativ im sehr abweichenden Individuenreichtum nicht selten überraschend aus. Brehm weiß davon bei der Besprechung der beiden durch einen Bach verbundenen Finstertalerseen im Sellrain zu berichten; Monti weist besonders deutlich auf die durch den Ursprung, die Geschichte und die mannigfaltigen äußeren Verhältnisse eines Sees bedingten Divergenzen der tierischen Bevölkerung hin. Aber auch Gunst und Ungunst aktiven oder passiven Tierimports in ein Hochseebecken spielen eine entscheidende Rolle bei der Gestaltung der Fauna. Szilády nennt für diese verschiedenen Faktoren treffende Beispiele aus seinem Untersuchungsgebiet, den transsylvanischen Alpen.

Stingelin konstatierte, daß in Jahren von ungleichem meteorologischem Charakter auch die Entomostrakenbevölkerung der Gotthardgewässer nach Zusammensetzung und Reichtum in weiten Grenzen variierte. Am reichsten an Crustaceen waren immer die warmen Torftümpel, am ärmsten die arktische Bedingungen bietenden Eisweiher. Zur Zeit des Eisbruchs, im Frühjahr der Hochalpen, erwies sich der Lago Lucendro, ein prächtiger Bergsee der Gotthardgruppe, als fast tierlos, später belebten sich Ufer und freies Wasser ausgiebig mit Entomostraken.

Szilády gelangt auf Grund seiner Untersuchungen zum Schluß, daß das Auftreten der einzelnen Arten von Crustaceen in hohem Grad unter dem Einfluß der physikalischen Bedingungen des Wohngewässers stehe. Er stellt in den transsylvanischen Alpen vier Typen stehender Gewässer mit je einer speziellen Krebsfauna auf. In den austrocknenden, tiefgelegenen Pfützen trüben Regenwassers entwickelt sich sehr rasch durch passiv hineingetragene Keime eine Gesellschaft nicht typischer Alpentiere. Besonders reich mit Crustaceen belebt sind lelumige Teiche auf den Matten des Hochplateaus in der Höhenlage $1600--1900 \mathrm{~m}$. Mit dem Glazialphänomen stehen in engem Zusammenhang die beiden letzten Kategorien von Gewässern, die Torfmoore und die eiszeitlichen, kalten Felsenteiche. 
Erstere liegen als alte Gletscherböden imnerhalb der Schneegrenze der Eiszeit. Auf die Zusammensetzung der Krebsfauna wirken entscheidend die Genese der Wohngewässer, ihre Lage, Bewachsung, Untergrundbeschaffenheit, Temperatur, Tiefe, die rhemische Zusammensetzung und die Bewegung des Wassers.

Bei ihren weitausgedehnten Studien an Gewässern der nach Süden gerichteten Alpenhänge kam R. Monti zur Uberzeugung, daß die Besiedlung der Hochgebirgsseen sich stufenweise vollziehe. Das Alter der Becken entscheidet in erster Linie mit über den Reichtum und den Charakter der Fauna. Zu diesem ersten Besiedlungsfaktor tritt in zweiter Linie der ganze Komplex äußerer Bedingungen, die jedes Seebecken besitzt. Physikalische und chemische Verhältnisse, Klima nnd Lage, der floristische Bestand des Ufers und Wassers, fördern oder verhindern die Entwicklung der aktiv eingedrungenen oder passiv eingeschleppten .Tiere und Tierkeime.

Neugebildete Seen, die eben durch den Rückzug des Gletschers freigelegt wurden, bleiben noch einige Zeit unbelebt. Dann stellen sich als erste Kolonisten die Diatomeen ein. Ihnen folgen später die Protozoen. Diese wiederum bereiten den Boden biologisch vor für eine einfache Uferfauna, zusammengesetzt aus Rhizopoden, Nematoden, Tardigraden und Entomostraken. Erst jetzt meldet sich tin an Arten und Individuen armes Plankton von Rädertierchen und Copepoden. Eine weitere Besiedlungsstufe bringt die reichere Entfaltung der pelagischen Welt und besonders das Auftreten der Cladoceren. Damit erst deckt sich der Tisch für die Fische.

An einer Reihe gut gewählter Beispiele erläutert Monti die allmählich fortschreitende Kolonisation hochgelegener Alpenseen. Sie legt Nachdruck darauf, daß die biologischen Vorbedingungen zuerst erfüllt sein müssen, bevor eine bestimmte Tiergruppe auftreten kann. Die Algen dienen den Rhizopoden als Nahrung, diese wiederum den Rotiferen. Die Rädertierchen sollen den Copepoden und letztere den Cladoceren zum Opfer fallen. Die aufgestellte Reihe des Fressens und Gefressenwerdens würde die Besiedlungsgeschichte der Hochgebirgsseen vornehmlich bestimmen.

Montis Darstellung enthält manches richtige. Es leuchtet ein, daß erst nachdem gewisse biologische Bestimmungell geschaffen sind, sich in einem See ein reicheres Plankton entfalten kamn oder Fische ihr Gedeihen finden können. Doch darf das aufgestellte Schema der stufenweisen Besiedlung nicht als strenge Regel gedacht werden, und auch die Annahme eimes in so einfacher systematischer Abstufung sich bewegenden Emährungszyklus hält einer Kritik wohl kaum stand.

Gerade Montis Beispiele zeigen, wie vor allem Gunst und Ungunst der Jokalität eine raschere oder weniger rasche Belebung eines alpinen Alpenbeckens bedingt, so daß in nächstgelegenen Seen eine sehr verschieden reiche und verschieden mannigfaltige Fauna sich entwickelt. Nachweisbar längst bestehende Bergseen bleiben tot, weil der Import von Tieren und Keimen sich nur schwer vollzieht und die eingeführten Lebewesen in ihnen ungünstige äuBere Bedingungen finden. Frischgebildete warme Almtümpel dagegen beleben sich wie auf einen Schlag mit einer bunten Gesellschaft cler verschiedensten Tiere.

Diese Almtümpel, denen die durch üppige Wasserblüte von Euglena sanguinea bedingte rote Farbe in der Schweiz den Namen "Blutseen" eingetragen hat, stellen im ganzen Alpengebiet eine biologisch ungemein gut charakterisierte Art stehender Gewässer dar. Starke Insolation erwärmt die nie ganz eintrocknenden Pfützen in kurzer Frist ausgiebig; aber anch die Abkühlung tritt unvermittelt bis zu tiefsten Temperaturgraden ein. Die Tümpel erlauben einer 
Gesellschaft von restistenten und eurythermen Tieren, zum Teil wenigstens Kosmopoliten, einen kräftigen Vorstoß hoch hinauf in die hochalpine Region. In jhnen herrscht Brachionus urceolaris, Anuraea valga, Mesostoma lingua und Daphnia pulex in Gesellschaft einiger gewöhnlicher Cyclopiden. Die Fauna bleibt gleich in den Zentralalpen der Schweiz, wie in Graubünden und Osterreich. Erst am Ostrand des mächtigen Gebirgssystems, in den Almtümpeln bei Lunz, tritt zu der gewöhnlichen Fauna, als östlicher Zuwanderer, Diaptomus tatricus. Uber die biologische und faunistische Bedeutung der Blutseen wird eine ausführliche Arbeit in dieser Zeitschrift nächstens sprechen.

Den Umstand, daß die kleinen und seichten Hochsem des Vigezzo- und Onsernonetals noch eine reiche tierische Bevölkerung beherbergen, trotzdem diese Becken den Kämmen und Gipfeln relativ naheliegen, möchte $R$. Monti gegen die Ansicht des Referenten anführen, nach welchen die kosmopolitische Fauna in weniger hohen und breiten Bergzügen auf tieferer Höhenstufe Halt macht, als in mächtigen und hochgetürmten Massiven. Sie übersieht dabei, daß Referent seinem Satz eine ganz allgemeine Fassung gab und unter Aufzählung von Beispielen auf die leicht $\mathrm{zu}$ erklärenden Ausnahmen aufmerksam machte.

Nach Monti übernahm vor allem passiver Tierimport durch Luftströmungen, fliegende Insekten und besonders durch weitziehende Vögel die Besiedelung der hochalpinen Gewässer nach dem RückfluB der Gletscher, Nur auf diesem Wege, führt die italienische Zoologin aus, konnten nordpolare, stenotherme Arten den Südhang der Alpen erreichen. Für aktive Wanderung nach dem Kamm und Gipfel sollen die reißenden Bergbäche keine geeigneten Straßen. bilden.

Dem ist entgegenzuhalten, daß gerade in den schäumenden Gebirgsbächen die meisten und am besten charakterisierten glazial-nordischen Relikte wohnen. Sie können in dem stürzenden Bach nur durch aktive Wanderung bergaufwärts gelangt sein. Der einstige Wasserreichtum der postglazialen Zeit öfnete dem aktiven Vordringen in die Höhe manche nun geschlossene und versiegte Straße. Manches Hindernis, das heute etwa als Wassersturz den Vormarsch hemmt, bestand damals noch nicht, oder konnte im Laufe der Jahrtausende, während welcher das Eis langsam, Zoll um Zoll zurückwich, geduldig überwunden worden. Alles scheint mir darauf hinzuweisen, dab das aktive, schrittweise Hinaufwandern durch die Adern der Bäche für die postglaziale Wiederbesiedelung hochalpiner Gewässer früher die höchste Bedeutung besab. Auch jetzt währt diese Wanderung nach oben noch weiter, in beschränktem Maße allerdings, denn der abnehmende Wasserreichtum und der sich steigernde Wildbachcharakter schließt manchen einstigen Bachbewohner und Wanderer aus den heutigen alpinen Wasserstraßen aus. Sogar in hochalpine Seen sind, nach Steinmann, torrenticole Planarien und Insektenlarven aktiv vorgedrungen.

Auch vor der Wasserscheide, die den Norden vom Süden auf dem Alpenkamm trennt, braucht die aktive Wanderung nicht Halt zu machen. Auf den Jochen und Sätteln der Gebirge verflechten sich die Quelläufe der nach verschiedenen Himmelsrichtungen fließenden Ströme. Aus einer Quelle entspringen nicht selten zwei Flüsse. Jedes alltägliche Ereignis, Lawinensturz, Bergrutsch, kann hydrographische Verschiebungen, Ablenkungen von Wasseradern nach anderen Flußsystemen mit sich bringen. In geologischen Zeiträumen verlängern sich, wie zahllose Beispiele lehren, Bachläufe nach rückwärts und nehmen so Nebenrinnsale auf, die früher anderen Flüssen zuströmten. Das alles aber öffnet neue und schlieBt alte Wasserstraßen und zeitigt so zunächst faunistische, in letzter Linie aber tiergeographische Folgen. Eine Fülle von Tatsachen der hochalpinen 
Tierverbreitung lassen sich nur durch die Annahme aktiver Tierwanderung erklären.

Andere Vorkommnisse allerdings finden ihre Deutung besser durch die Hypothese der passiven Verschleppung. Ihr habe ich denn auch, das sei gegenüber R. Monti ausdrücklich betont, eine große Rolle besonders für die Einfuhr ubiquistischer Elemente in die pelagische und littorale Region der Hochalpenseen zugeschrieben. Monti selbst macht übrigens darauf aufmerksam, daB das Vorkommen und Fehlen von Daphnien in nächstgelegenen, durch Bäche verbundenen Wasseransammlungen gegen die Leichtigkeit passiven Transportes spreche.

In bemerkenswerter Ausführung entscheidet sich auch Brehm für die Annahme aktiver Einwanderung mancher nordischer Elemente in die Wasserfauna der Alpenseen; die Bedeutung der passiven Einschleppung durch Wasservögel hält er für stark überschätzt. Immerhin schließt der genannte Planktonforscher Organismen, wie pelagische Copepoden, von der aktiven Wanderung in Gebirgsbächen aus. Dem wird zuzustimmen sein.

Wenn sich so über die Zusammensetzung der hochalpinen Fauna und über die Besiedelungsgeschichte der Hochgebirgsseen mancherlei Licht verbreitet, hat sich das Dunkel über den biologischen Problemen kaum erhellt.

Der Beantwortung harrt fast noch ganz die wichtige Frage nach dem Gang der lokalen und temporalen Variation der Cladoceren im Hochgebirge. Einem. jungen Forscher würde sich gerade hicr ein lohnendes Feld der Beobachtung bieten und zugleich die Aussicht öffnen, den letzten Ursachen der Variationen durch Vergleichung der im Norden, im europäischen Flachland und in den Alpen gewonnenen Erfahrungen erfolgreich nachzuspüren.

Im allgemeinen läßt sich vielleicht erwarten, daß der Cyclomorphose in den Bergseen, wie dem Gang der Temperatur und der Veränderung der inneren Reibung des Wassers enge Grenzen gezogen seien.

Von der in den Seen und Tümpeln der hochalpinen Region so regelmäßig auftretenden Daphnia longispina weiß Stingelin nach seinen Erfahrungen auf dem St. Gotthard zu berichten, daß die Formveränderungen sich in den Variationsrichtungen bewegen, die Ekman und Lilljeborg in den skandinavischen Gebirgen beobachteten. Am weitesten verbreitet ist im Gotthardgebiet ein der Var. rosea von D. longispina nahestehender Typus; in einem Torftümpel erschien auch die Var. abbreviata Lilljeb. der schwedischen Hochgebirge. Im Laj Nair des Unterengadins dagegen lebt die in der Schweiz sonst unbekannte D. longispina var. tenuiseta Sars.

Besonders typisch aber für die Hochalpen scheint das Auftreten großer, plumper Daphnien zu sein, die an nordische Formen lebhaft erinnern. Schon früher beschrieb Stingelin aus einem Hochsee des St. Bernhard D. Zschokkei, die sich später auch in Finnland und Skandinavien fand. Von dieser Art verbreitet sich die durch Größe ausgezeichnete Var. vigezzina Monti in den Hochseen des südlichen Alpenabfalls. R. Monti fand den Krebs in typischer Form sowohl im Lae d'Arpy des Ruitor $(2050 \mathrm{~m})$, als in den Alpenseen des Vigezzound Onsernonetals und im Tosa- und Aostagebiet. Sie betrachtet das Tier als konstante Lokalvariation von D. Zschokkei, die in den morphologischen Jahreszyklus von D. Iongispina um so weniger eingeschoben werden dürfe, als $D$. longispina und D. Zschokkei-vigezzina gleichzeitig in demselben Seebecken leben können.

Besondere Beachtung verdient die durch Szilády mitgeteilte Tatsache, daß D. Zschokkei auch in den höchstgelegenen Gebirgsseen des Retyezat bei 2100 
bis $2200 \mathrm{~m}$ lebt. In Torfgewässern der transsylvanischen Alpen tritt an ihre Stelle D. alpina Dad, die mit D. obtusa verwandt aber nicht identisch sein soll. Glaziale Wasseransammlungen des Retyezat endlich beherbergen D. longispina var. Leydigi.

Morphologische und biologische Erwägungen lassen Ekman einen engen genetischen Zusammenhang zwischen der skandinavischen Daphnia longispina var. abbreviata und der hochalpinen D. Zschokkei als sehr wahrscheinlich vermuten. So spannt sich auch hier zwischen dem Norden und dem mitteleuropäischen Gebirge wieder ein tiergeographisches Band aus.

Als Daphnie der Dolomitenseen hat, nach Brehm, die Forma Foreli von D. Iongispina der microcephala-Reihe zu gelten.

Der Variation dürfte bei den Hochgebirgsdaphnien auch der Bau des Auges unterliegen. Wenigstens deuten die vorläufigen Bemerkungen Wolterecks darauf hin, daß bei D. pulex der warmen Almtümpel eine Vermehrung des Augenpigments und der Linsenzahl sich einstelle.

In allen diesen Einzeldaten liegt der Hinweis darauf, daß das Studium der alpinen Daphniden für die Auffassung von der Entstehung der Varietäten fruchtbringend werden müsste, besonders wenn mit der Beobachtung in der Natur das kontrollierende Experiment Hand in Hand gehen könnte. Daneben würden sich wertvolle, tiergeographische Resultate gewinnen lassen.

In beiden Richtungen liefern auch andere Cladoceren des Hochgebirgs treffliches Material. Stingelin weist auf die ungezählten Variationen hin, welche die eine Art Chydorus sphaericus allein in den so verschiedenartigen Gewässern der Grotthardgruppe in Form, Farbe und Schalenstruktur eingeht. Derselbe Autor unterscheidet in unmittelbar nebeneinander liegenden Wasseransammlungen des St. Gotthard zwei deutlich differente Formen des nordischen Holopedium gibberum. In gröberen, durchsichtigen Seen bleibt die Cladocere klein, farbloshyalin. Dieser pelagischen Form läßt sich das größere, gelbe oder gelbbraune Holopedium pflanzendurchwachsener Tümpel entgegenstellen, das gleichzeitig viel mehr Eier erzeugt, als die den See bewohnenden Artgenossen. Immerhin bleiben auch die Holopedien der Tümpel an Größe und Eizahl noch weit hinter den Speziesvertretern des hohen Nordens zurück. Dies deutet, wie in ähnlichen, nicht seltenen Parallelfällen (Polyphemus, Diaptomus denticornis), wieder auf eine nordisch-glaziale Urheimat der Spezies hin.

Beginnende Rassenbildung vermutet Brehm bei der im Lichtsee $(2200 \mathrm{~m})$ isolierten Kolonie von Diaptomus denticornis.

Der hintere Finsterthaler See und der Wildsee am Wildloder lieferte Brehm stachellose oder sehr kurz bestachelte Varietäten von Anuraea aculeata.

Als eine biologische Anpassung an das Leben in wenig tiefen bis zum Grund durchsichtigen Hochalpenseen darf es betrachtet werden, wenn das Plankton in horizontaler Wanderung die im Schatten liegenden Abschnitte des Wohngewässers aufsucht, R. Monti beobachtete diese Erscheinung in überraschender Weise am Lago di Panelatte $(2048 \mathrm{~m})$, dessen freischwimmende Organismenwelt zum guten Teil aus der hochrot gefärbten Heterocope saliens besteht. Die Lichtflucht führt oft die gesamte tierische Planktonmenge in durch Bergwände oder Felsen vor den direkten Sonnenstrahlen geschützte Seeteile zusammen. So tritt an die Stelle vertikalen Abstiegs für pelagische Organismen in hochalpinen Seen horizontale nach Tageszeit und Besonnung sich regelnde Verschiebung.

Auch Brehm faßt nach seinen Erfahrungen am Achensee die Wanderungen der Planktozoen als eine Äußerung von Phototropismus auf. Doch ist für ihn, 
wie für Ekman, die Leukophobie eine sekundär erworbene Eigenschaft, die sich durch die Gewohnheit der kältebedürftigen, nordischen Plantontiere ausbildete, die niedrig temperierten dunkeln Seetiefen während des Tages anfzusuchen. Primär wäre die nordische Stenothermie, später erworben die Lichtscheu. Um seine Ansicht, daß die Temperatur in letzter Linie Planktonwanderungen bedinge, zu stützen, beruft sich Brehm u. a. auf die Beobachtung, daB exklusive Tiefenbewohner der großen subalpinen Seen am Ufer der Hochgebirgsbecken ihr Leben fristen können. Auch in diesem Fall würde die Temperatur und nicht das Licht die vertikale Verteilung der Süßwassertiere beherrschen.

Gerade aus den faunistischen Listen R. Montis ergeben sich hübsche neue Beispiele für die Ubereinstimmung der profunden Fauna der subalpinen Randseen und der littoralen Tierwelt kleiner Hochgebirgsbecken. Im Lac de Ruitor $(2602 \mathrm{~m})$ lebt Pseusodifflugia archeri, im Lac gris $(2600 \mathrm{~m})$ Hyalosphenia punctata, im Lac vert $(2600 \mathrm{~m})$ Nebela vitraea und endlich im Lac de Pierre rouge und im Lac d'Arpy $(2550$ und $2050 \mathrm{~m})$ Cyphorleria calceolus. Diese vier Rhizopoden des Ruitorgebiets aber fehlen dem Flachwasser der Ebene; sie gehören dagegen zu jener typischen Protozoen-Fauna, die Penard in den großen Tiefen des Genfersees und anderer subalpiner Becken der Schweiz antraf. So öfnet sich ein neuer Blick auf den gemeinsamen Urprung der tierischen Bevölkerung von hochappinen Weihern und der Tiefenfaund dor Seen des Flachlands aus der stenothermen Tierwelt des postglazialen Schmelzwassers. R. Monti selbst übersah die Cbereinstimmung der Rhizopoden seheinbar so verschiedener und weit anseinander liegender Wohnorte.

Die grelle Karotinfärbung der hochalpinen Copepoden deutet Brehm als ein Schutzmittel gegen die Kälte, indem die roten Farbstoffe die Fähigkeit besitzen sollen, Licht in Wärme umzusetzen. Dje Lösung der Frage nach der Bedeutung der hotfärbung steht noch aus; alle neueren Untersuchungen an Hochgebirgsgewïssern brachten nur die vielfache Bestätigung der bekannten Tatsache, daß die rote Farbe der Entomostraken die weiteste Verbreitung besitzt und daß ihre Intensität mit der sinkenden Temperatur der Wohngewässer sich im allgemeinen steigert.

Einen Fall von Veränderung der Emährungsweise beobachtete Steinmann an der sonst karnivoren Planaria alpina, die im Partnunersee Algenkost nicht zu verschmähen scheint.

Eine weite Lücke endlich weisen unsere Kenntnisse vom Jahreszyklus der horhalpinen Entomostraken auf. Uber den Eintritt der Dauereibildung liegen nur vereinzelte zusammenhanglose Daten vor. So bleibt leider der Wunsch unerfüllt, in dieser Beziehung eine biologische Vergleichung zwischen den Hochalpen und ‘lem Norden durchzuführen. Die Zukunft wird im zentral-europäischen Gebirge das nötige Parallelmaterial zu den glänzenden Untersuchungen skandinavischer Zoologen, besonders Ekmans, sammeln müssen. Erst auf die biologische Vergleichung gestützt, werden sich vollgültige Schlüsse über die Herkunft eines großen Teils der Süßwasserfauna Mitteleuropas ziehen lassen.

Keilhack fand Mitte Juni in einem von Schnee bedeckten Hochalpensee der Dauphiné nur Dauereier von Cladoceren. Das aktive Leben war noch nicht erwacht. Stingelin verzeichnet dasselbe Faktum für das hochgelegene Eisbecken des Lago dell' uomo auf dem St. Gotthard noch im August $(2445 \mathrm{~m})$. Es handelte sich in dem letztgenannten Fall um D. longispina; die Art bildete in einem wärmeren Jahre in demselben Wasserbecken im August Danereier.

Bei einer Temperatur von $6^{\circ} \mathrm{C}$ enthielt der Gotthardsee Lago Orsirora 
(2456 m) im August nur Ephippien von Daphnia longispina, Alona affinis und Alonella excisa. Letztere Form erzeugte im Lago di Sella im Jahr 1895 die Dauereier Ende August. Daphnia hyalina sieht sich im alljährlich eintrocknenden Dürrensee gezwungen, im Hochsommer regelmäßig Dauereier zu erzeugen.

Holopedium gibberum der Gotthardseen sieht Stingelin als monozyklisch an. Weder im Juli noch im August beobachtete er Männchen, oder mit Dauereiern beladene Weibchen.

Die vorausgehende Zusammenstellung zeigt, einen wie breiten Raum rein faunistische Beobachtungen an Hochalpenseen immer noch einnehmen. Sie läßt aber auch erkennen, welche wichtigen tiergeographischen und biologischen Fragen an den Gebirgsgewässern der Lösung harren. Die Anfänge zur Behandlung djeser tiefergreifenden Probleme liegen vor, und die zu beschreitenden Wege sind ausgesteckt vor allem durch die Untersuchungen Steuers an der alten Donau bei Wien und Ekmans in den nordschwedischen Hochgebirgen. Vergleichung der Lebensweise der arktischen Süßwasserbewohner mit derjenigen der postglazial in den Alpen zurückgebliebenen Reliktenkolonien muß als eines der nächsten Ziele zoologischer Erforschung der Hochgebirgsseen gelten. 